\title{
SOLVABILITY OF QUASILINEAR ELLIPTIC EQUATIONS WITH NONLINEAR BOUNDARY CONDITIONS
}

\author{
BY
}

GARY M. LIEBERMAN

ABSTRACT. On an $n$-dimensional domain $\Omega$, we consider the boundary value problem

$$
Q u=0 \quad \text { in } \Omega, \quad N u=0 \text { on } \partial \Omega
$$

where $Q$ is a quasilinear elliptic second-order differential operator and $N$ is a nonlinear first order differential operator satisfying an Agmon-Douglis-Nirenberg consistency condition. If the coefficients of $Q$ and $N$ satisfy additional hypotheses (such as sufficient smoothness), Fiorenza was able to reduce the solvability of $(*)$ to the establishment of a priori bounds for solutions of a related family of boundary value problems. We simplify Fiorenza's argument, obtaining the reduction under more general hypotheses and requiring a priori bounds only for solutions of $Q u=f$, $N u=g$ where $f$ and $g$ range over suitable function spaces. As an example, classical solutions of the capillary problem are shown to exist without using the method of elliptic regularization.

0. Introduction. Suppose that $Q u=a^{i j}(x, u, D u) D_{i j} u+a(x, u, D u)$ is a quasilinear operator on a domain $\Omega \subset \mathbf{R}^{n}$ and that $N u=b(x, u, D u)$ is a nonlinear operator on $\partial \Omega$. (Here and in what follows, the convention that repeated indices are to be summed from 1 to $n$ is observed.) Under suitable ellipticity and related conditions on $Q$ and $N$, Fiorenza [5] was able to reduce the solvability of the problem

$$
Q u=0 \quad \text { in } \Omega, \quad N u=0 \text { on } \partial \Omega
$$

to the establishment of certain a priori bounds via a theorem of Caccioppoli $[11, \S 41 . I I I]$. Specifically (see [9, Chapter 10]), all solutions of

$$
Q_{t} u=0 \quad \text { in } \Omega, \quad N_{t} u=0 \quad \text { on } \partial \Omega
$$

must be bounded in some Banach space (typically $C^{1+\alpha}(\bar{\Omega})$ ) uniformly with respect to $t$ and $u$ for some appropriate family of pairs of operators $\left(Q_{t}, N_{t}\right)_{t \in[0,1]}$. For Dirichlet boundary conditions, i.e. $b(x, u, D u) \equiv u$, this reduction is achieved under very general conditions via the Leray-Schauder theorem (see, e.g., $[7,9,12])$; however for more general boundary conditions, several complications arise in the reduction. In this work we address ourselves to two of these.

First, Fiorenza used Schauder-type estimates (for related linear equations) which require explicit knowledge of their dependence on the coefficients. In $§ 2$, we show that simpler estimates may be used.

Received by the editors April 20, 1981 and, in revised form, September 4, 1981.

1980 Mathematics Subject Classification. Primary 35J25, 35 J65.

Key words and phrases. Quasilinear elliptic equations, nonlinear boundary conditions, capillary problem, fixed point theorem. 
Second, Caccioppoli's theorem requires that all problems $(0.2)$ be uniquely solvable for each $t$ whereas the Leray-Schauder theorem does not require this uniqueness. In §3, we shall use essentially a theorem of Kirk and Caristi to modify this assumption.

1. Notations and definitions. We shall use the following norms and seminorms:

$$
\begin{aligned}
& |u|_{0 ; \Omega}=\sup _{\Omega}|u|, \\
& {[u]_{\alpha ; \Omega}=\sup \left\{|u(x)-u(y)| /|x-y|^{\alpha}: x \neq y, x, y \text { in } \Omega\right\},} \\
& |u|_{\alpha ; \Omega}=|u|_{0 ; \Omega}+[u]_{\alpha ; \Omega},|u|_{1 ; \Omega}=|u|_{0 ; \Omega}+|D u|_{0 ; \Omega}, \text { and so on. Here } 0<\alpha<1,
\end{aligned}
$$

$D u$ is the gradient of $u$, and $\Omega \subset \mathbf{R}^{n}$ is a domain. If also $\partial \Omega$ is sufficiently smooth, say $\partial \Omega \in C^{2+\alpha}$, these norms can also be defined on $\partial \Omega$. We shall always assume

$$
\Omega \subset \mathbf{R}^{n}, n \geqslant 2 \text {, is a domain with } \partial \Omega \in C^{2+\alpha} \text {. }
$$

The inner normal to $\partial \Omega$ at $x$ will be denoted $\gamma(x)$ or just $\gamma$. Its components are $\gamma_{1}, \ldots, \gamma_{n}$.

For $K>0$, we define

$$
\begin{aligned}
\Omega(K) & =\left\{(x, z, p) \in \Omega \times \mathbf{R} \times \mathbf{R}^{n}:|z|+|p|<K\right\} . \\
\partial \Omega(K) & =\left\{(x, z, p) \in \partial \Omega \times \mathbf{R} \times \mathbf{R}^{n}:|z|+|p|<K\right\} .
\end{aligned}
$$

We shall also use the following abbreviations for derivatives: Let $g=g(x, z, p, r)$ be defined on $\Omega \times \mathbf{R} \times \mathbf{R}^{n} \times \mathbf{R}^{n^{2}}$. Then

$$
g_{x}=\left(\frac{\partial g}{\partial x^{1}}, \ldots, \frac{\partial g}{\partial x^{n}}\right), \quad g_{z}=\frac{\partial g}{\partial z}, \quad g_{p}=\left(\frac{\partial g}{\partial p_{1}}, \ldots, \frac{\partial g}{\partial p_{n}}\right) .
$$

If $G u=g\left(x, u, D u, D^{2} u\right)$, then

$$
G^{\prime}[u ; x]=\left(G^{\prime}\left[u ; x^{1}\right], \ldots, G^{\prime}\left[u ; x^{n}\right]\right)=g_{x}\left(x, u, D u, D^{2} u\right) .
$$

$G^{\prime}[u ; z]$ and $G^{\prime}[u ; p]$ are defined similarly. For example, if

$$
Q u=a^{i j}(x, u, D u) D_{i j} u+a(x, u, D u),
$$

then

$$
Q^{\prime}\left[u ; p_{k}\right]=\frac{\partial a^{i j}}{\partial p_{k}}(x, u, D u) D_{i j} u+\frac{\partial a}{\partial p_{k}}(x, u, D u) .
$$

If $g$ is defined on $\Omega(K)$ and $\beta \geqslant 0$, we denote by $|g|_{\beta ; K}$ the norm $|g|_{\beta ; \Omega(R)}, \Omega(K)$ being considered as a subset of $\mathbf{R}^{2 n+1}$. In particular if $g$ depends only on $x$ and $z$, then

$$
|g|_{0 ; K}=\sup \{g(x, z): x \in \Omega,|z|<K\},
$$

and similarly for $|g|_{\beta ; K}$ where $\beta>0$. Moreover if $g$ is defined only on $\partial \Omega(K)$, we also denote by $|g|_{\beta ; K}$ the norm $|g|_{\beta ; \partial \Omega(K)}$. (By assumption (1.1), this norm can be defined.)

Finally we shall denote a matrix $\left(a^{i j}\right)$ by the corresponding script letter $\mathcal{Q}$, a vector $\left(a^{i}\right)$ by $a$, and the sum $a^{i} b_{i}$ by $a \cdot b$. 
2. Schauder estimates and convergence results. In [7, Theorem 6.30] a Schauder estimate for the linear oblique derivative problem is given. A slight modification of the proof of this theorem yields

LemMA 1. Let $L u=a^{i j}(x) D_{i j} u$ in $\Omega, M u=\beta^{i}(x) D_{i} u(x)$. Suppose that

$$
\begin{gathered}
|\mathscr{Q}|_{\alpha}+|\beta|_{\alpha} \leqslant \Lambda, \\
\left|\beta^{i}(x) \gamma_{i}(x)\right| \geqslant \lambda \quad \text { on } \partial \Omega, \quad a^{i j}(x) \xi_{i} \xi_{j} \geqslant \lambda|\xi|^{2} \quad \text { for } x \in \Omega, \xi \in \mathbf{R}^{n}
\end{gathered}
$$

where $\alpha, \lambda, \Lambda$ are positive constants with $\alpha<1$. Then any solution $u \in C^{2+\alpha}(\bar{\Omega})$ of $L u=f$ in $\Omega, M u=\varphi$ on $\partial \Omega$ satisfies the estimate

$$
|u|_{2+\alpha} \leqslant C(\alpha, \lambda, \Lambda, n, \Omega)\left(|u|_{0}+|\varphi|_{1+\alpha}+|f|_{\alpha}+|D u|_{0} \cdot[\beta]_{1+\alpha}\right) \text {. }
$$

REMARK. (1) This estimate is similar to Fiorenza's [5, (5.2)].

(2) In fact the constant $C$ depends on $\beta$ only through $|\beta|_{0}, \lambda$, and a modulus of continuity for $\beta$.

From this estimate, we obtain our basic result on convergence of solutions for the nonlinear oblique derivative problem.

\section{Lemma 2. Define}

$$
Q u=a^{i j}(x, u, D u) D_{i j} u+a(x, u, D u), \quad N u=b(x, u, D u),
$$

and suppose that

$$
\begin{aligned}
& a^{i j} \xi_{i} \xi_{j} \geqslant \lambda|\xi|^{2}, \quad b_{p} \cdot \gamma \geqslant \lambda \quad \text { for }(x, z, p) \in \Omega(K), \xi \in \mathbf{R}^{n}, \\
& |\mathfrak{Q}|_{\alpha ; K}+|a|_{\alpha ; K} \leqslant \Lambda \text {, } \\
& \left|b_{p}\right|_{1+\alpha ; K}+|b|_{1+\alpha ; K} \leqslant \Lambda \text {, }
\end{aligned}
$$

where $\alpha, K, \lambda, \Lambda$ are positive constants with $\alpha<1$. Suppose also that for each positive integer $m$, there are functions $u_{m} \in C^{2+\alpha}(\bar{\Omega}), f_{m} \in C^{\alpha}(\bar{\Omega}), \varphi_{m} \in C^{1+\alpha}(\partial \Omega)$ such that

$$
\begin{gathered}
Q u_{m}=f_{m} \quad \text { in } \Omega, \quad N u_{m}=\varphi_{m} \text { on } \partial \Omega, \\
\left|f_{m}\right|_{\alpha}+\left|\varphi_{m}\right|_{1+\alpha} \leqslant K_{1},
\end{gathered}
$$

the sequences $\left(f_{m}\right),\left(\varphi_{m}\right),\left(u_{m}\right)$ converge uniformly to $f, \varphi, u$ respectively,

where $K_{1}$ is a constant independent of $m$. Suppose finally that

$$
\left|u_{m}\right|_{1} \leqslant K, \quad\left|u_{m}\right|_{1+\delta} \leqslant K_{2} \text { for some } \delta \in(0,1)
$$

where $K_{2}>0$ is a constant and $K$ is the constant in (2.2). Then $u \in C^{2+\alpha}(\bar{\Omega})$ and

$$
Q u=f \quad \text { in } \Omega, \quad N u=\varphi \text { on } \partial \Omega \text {. }
$$

REMARK. Clearly $f \in C^{\alpha}(\bar{\Omega}), \varphi \in C^{1+\alpha}(\partial \Omega), \varphi_{m} \rightarrow \varphi$ in $C^{1}(\partial \Omega)$, and $u_{m} \rightarrow u$ in $C^{1}(\bar{\Omega})$.

Proof. Let $v=u_{m}-u_{k}$ where $m, k$ are positive integers. Then $v$ is a solution of the linear problem

$$
L v=g \quad \text { in } \Omega, \quad M v=\psi \text { on } \partial \Omega
$$


where

$$
\begin{gathered}
L u=\bar{a}^{i j} D_{i j} u, \quad M u=\beta^{i} D_{i} u, \\
\bar{Q}=\mathbb{Q}\left(x, u_{m}, D u_{m}\right), \quad \beta=\int_{0}^{1} b_{p}\left(x, u_{m}, D u_{k}+t\left(D u_{m}-D u_{k}\right)\right) d t, \\
g=\left(f_{m}-f_{k}\right)+\left(a\left(x, u_{k}, D u_{k}\right)-a\left(x, u_{m}, D u_{m}\right)\right) \\
+\left(a^{i j}\left(x, u_{k}, D u_{k}\right)-a^{i j}\left(x, u_{m}, D u_{m}\right)\right) D_{i j} u_{k}, \\
\psi=\left(\varphi_{m}-\varphi_{k}\right)+\left(b\left(x, u_{k}, D u_{k}\right)-b\left(x, u_{m}, D u_{k}\right)\right) . \\
|g|_{\alpha \delta} \leqslant \varepsilon(m, k)\left(1+K_{3}+K_{4}\right)+c_{1} K_{3}, \quad|\psi|_{1+\alpha \delta} \leqslant \varepsilon(m, k)\left(1+K_{3}+K_{4}\right), \\
|\beta|_{\alpha \delta} \leqslant c_{1}, \quad[\beta]_{1+\alpha \delta} \leqslant c_{1}\left(1+K_{3}+K_{4}\right)
\end{gathered}
$$

where $\varepsilon(m, k)$ is a constant depending on $\alpha, \delta, k, K, K_{1}, K_{2}, \Lambda, m, \Omega$ such that

$$
\begin{gathered}
\varepsilon(m, k) \rightarrow 0 \text { as } m, k \rightarrow \infty, \\
K_{3}=\max \left\{\left|D^{2} u_{m}\right|_{0},\left|D^{2} u_{k}\right|_{0}\right\}, \quad K_{4}=\max \left\{\left[D^{2} u_{m}\right]_{\alpha \delta},\left[D^{2} u_{k}\right]_{\alpha \delta}\right\},
\end{gathered}
$$

and $c_{1}$ is a constant depending only on $K, K_{2}, \Lambda$. Lemma 1 then applies giving

$$
[v]_{2+\alpha \delta} \leqslant c_{2}\left(1+K_{3}\right)+\varepsilon^{\prime}(m, k) K_{4}
$$

where $c_{2}$ depends on $\alpha, \delta, K, K_{1}, K_{2}, \Lambda$, and $\Omega, \varepsilon^{\prime}$ depends on these quantities as well as $m$ and $k$, and $\varepsilon^{\prime}$ satisfies (2.6).

We now apply the well-known interpolation inequality (see [7, Theorem 6.35] or $[11, \S 33])$ :

$$
\left|D^{2} u\right|_{0} \leqslant c_{3} \eta^{-2 /(\alpha \delta)}|u|_{0}+\eta[u]_{2+\alpha \delta},
$$

where $c_{3}$ depends only on $\alpha \delta$ and $\Omega$, with $\eta=\left(4 c_{2}+4\right)^{-1}$, to obtain

$$
[v]_{2+\alpha \delta} \leqslant c_{4}+\left(\frac{1}{4}+\varepsilon^{\prime}(m, k)\right) K_{4}
$$

where $c_{4}$ depends on the same quantities as $c_{2}$ and $\varepsilon^{\prime}$ is the same as in (2.7).

Hence $\left[u_{m}\right]_{2+\alpha \delta}$ is bounded independent of $m$. For suppose not; then

$$
K_{4}\left[u_{m}\right]_{2+\alpha \delta}>4\left[u_{k}\right]_{2+\alpha \delta} \geqslant 4 c_{4}, \quad \varepsilon^{\prime}(m, k) \leqslant \frac{1}{4}
$$

for some $m$ and $k$. Hence $[v]_{2+\alpha \delta} \geqslant \frac{3}{4} K_{4}$. Therefore (2.8) implies that $\frac{3}{4} K_{4} \leqslant c_{4}$ $+\frac{1}{2} K_{4}$ so that $K_{4} \leqslant 4 c_{4}$, contradicting (2.9). Thus $\left[u_{m}\right]_{2+\alpha \delta}$ is bounded independent of $m$. Therefore $u_{m} \rightarrow u$ in $C^{2}(\bar{\Omega})$ so that (2.5) is valid.

To complete the proof, we note that

$$
|g|_{\alpha}+|\psi|_{1+\alpha} \leqslant c_{5}+\varepsilon(m, k) K_{5}, \quad|\beta|_{\alpha} \leqslant c_{5}, \quad[\beta]_{1+\alpha} \leqslant c_{5}\left(1+K_{5}\right)
$$

where $c_{5}$ depends on $\alpha, \delta, K, K_{1}, K_{2}, K_{3}, K_{4}, \lambda, \Lambda$, and $\Omega, \varepsilon$ depends on these quantities as well as $m$ and $k,(2.6)$ holds, and $K_{5}=\max \left\{\left[u_{m}\right]_{2+\alpha},\left[u_{k}\right]_{2+\alpha}\right\}$. We then conclude (as from (2.8)) that $\left[u_{m}\right]_{2+\alpha}$ is bounded independent of $m$.

REMARKS. (1) Our proof is a modification of Fiorenza's in [5].

(2) Under stronger hypotheses on $\mathbb{Q}$ and $a$, Fiorenza showed that

$$
[v]_{2+\alpha \delta} \leqslant c_{6}\left(1+K_{4}\right)\left(|v|_{1}+\left|f_{m}-f_{k}\right|_{\alpha \delta}+\left|\varphi_{m}-\varphi_{k}\right|_{1+\alpha \delta}\right) .
$$


Hence, if $f_{m} \rightarrow f$ in $C^{\alpha}(\bar{\Omega})$ and $\varphi_{m} \rightarrow \varphi$ in $C^{1+\alpha}(\delta \Omega)$ then $u_{m} \rightarrow u$ in $C^{2+\alpha \delta}(\bar{\Omega})$. Although it is not noted there, it also follows that $u_{m} \rightarrow u$ in $C^{2+\alpha}(\bar{\Omega})$.

From the proof of Lemma 2 we see that in fact a stronger result, which has not been noted before, is valid.

COROLlaRy 1. For each positive integer $m$, define

$$
Q_{m} u=a_{m}^{i j}(x, u, D u) D_{i j} u+a_{m}(x, u, D u), \quad N_{m} u=b_{m}(x, u, D u)
$$

and suppose that $Q_{m}, a_{m}, b_{m}$ satisfy (2.2) with $\alpha, K, \lambda, \Lambda$ independent of $m$. Suppose also that there are functions $u_{m}, f_{m}, \varphi_{m}$ as in Lemma 2 such that

$$
Q_{m} u_{m}=f_{m} \quad \text { in } \Omega, \quad N_{m} u_{m}=\varphi_{m} \quad \text { on } \partial \Omega
$$

and (2.3b), (2.3c), (2.4) hold. Suppose finally that $Q_{m}, a_{m}, b_{m}$ converge uniformly (on $\Omega(K))$ to $\mathcal{Q}, a, b$, respectively. Then $u \in C^{2+\alpha}(\bar{\Omega})$, and $u$ satisfies $(2.6)$ where $Q$ and $N$ are given by (2.1).

Proof. The same as Lemma 2 except that now $\overline{\mathcal{Q}}, g$, and $\psi$ given by

$$
\begin{aligned}
& \overline{\mathbb{Q}}=\mathbb{Q}_{m}, \quad g=\left(f_{m}-f_{k}\right)+\left(a_{k}-a_{m}\right)+\left(a_{k}^{i j}-a_{m}^{i j}\right) D_{i j} u_{k}, \\
& \psi=\left(\varphi_{m}-\varphi_{k}\right)+\left(b\left(x, u_{k}, D u_{k}\right)-b\left(x, u_{m}, D u_{k}\right)\right)+\psi_{m}-\psi_{k}
\end{aligned}
$$

where $\psi_{m}=b_{m}-b\left(x, u_{m} D u_{m}\right)$ and $Q_{m}, a_{m}, b_{m}$ are evaluated at $\left(x, u_{m}, D u_{m}\right)$. A straightforward calculation then shows that

$$
\begin{aligned}
& \left|\psi_{m}\right|_{\alpha \delta} \leqslant c_{1}, \quad\left[\psi_{m}\right]_{1+\alpha \delta} \leqslant c_{1}\left(1+\left|D^{2} u_{m}\right|_{0}\right)+\varepsilon(m)\left[u_{m}\right]_{2+\alpha \delta}, \\
& \left|\psi_{m}\right|_{\alpha} \leqslant c_{2}, \quad\left[\psi_{m}\right]_{1+\alpha} \leqslant c_{2}+\varepsilon^{\prime}(m)\left[u_{m}\right]_{2+\alpha},
\end{aligned}
$$

where $c_{1}$ depends on $\alpha, \delta, K, K_{2}$, and $\Omega, \varepsilon$ depends on $m$ and the same quantities as $c_{1}, c_{2}$ depends on $K_{3}$ and the same quantities as $c_{1}, \varepsilon^{\prime}$ depends on $m$ and the same quantities as $c_{2}, K_{3}$ is defined as in Lemma 2, and $\varepsilon, \varepsilon^{\prime}$ tend to 0 as $m \rightarrow \infty$.

Another convergence result for operators with smoother coefficients also follows.

Corollary 2. Define $Q$ and $N$ by (2.1) and suppose (2.2a) and

$$
|\mathbb{Q}|_{1+\alpha ; K}+|a|_{1+\alpha ; K}+|b|_{2+\alpha ; K} \leqslant \Lambda
$$

where $\alpha, K, \lambda, \Lambda$ are positive constants with $\alpha<1$. Suppose that $\partial \Omega \in C^{3+\alpha}$ and that for each positive integer $m$, there are functions $u_{m} \in C^{3+\alpha}(\bar{\Omega}), f_{m} \in C^{1+\alpha}(\bar{\Omega}), \varphi_{m} \in$ $C^{2+\alpha}(\partial \Omega)$ satisfying (2.3a), (2.3c), (2.4) and $\left|f_{m}\right|_{1+\alpha}+\left|\varphi_{m}\right|_{2+\alpha} \leqslant K_{1}$. Then $u \in$ $C^{3+\alpha}(\bar{\Omega})$ and $u$ is a solution of (2.5).

Proof. By virtue of Lemma 2, $u \in C^{2+\alpha}(\bar{\Omega}), u$ is a solution of (2.5), and $\left|u_{m}\right|_{2+\alpha}$ is bounded independent of $m$.

We now extend $\gamma$ to all of $\Omega$ so that $\gamma \in C^{2+\alpha}(\bar{\Omega})$ and $|\gamma| \leqslant 1$ in $\Omega$. Noting that the operator $c^{r k} D_{k}$, where $c^{r k}=\delta^{r k}-\gamma_{r} \gamma_{k}$, is a tangential operator on $\partial \Omega$, we have that $v=c^{r k} D_{k} u_{m}$ is a solution of

$$
L v=g \quad \text { in } \Omega, \quad M v=\psi \quad \text { on } \partial \Omega .
$$


Here

$$
\begin{gathered}
L v=\bar{a}^{i j} D_{i j} v, \quad M v=\beta^{i} D_{i} v, \\
\bar{Q}=Q \mathcal{Q}\left(x, u_{m}, D u_{m}\right), \quad \beta=N^{\prime}\left[u_{m} ; p\right], \\
g=c^{r k}\left\{D_{k} f_{m}-Q^{\prime}\left[u_{m} ; x^{k}\right]-Q^{\prime}\left[u_{m} ; z\right] D_{k} u_{m}-Q^{\prime}\left[u_{m} ; p_{i}\right] D_{i k} u_{m}\right\} \\
+2 \bar{a}^{i j} D_{i}\left(c^{r k}\right) D_{j k} u_{m}+\bar{a}^{i j} D_{i j}\left(c^{r k}\right) D_{k} u_{m}, \\
\psi=c^{r k}\left\{D_{k} \varphi_{m}-N^{\prime}\left[u_{m} ; x^{k}\right]-N^{\prime}\left[u_{n} ; z\right] D_{k} u_{m}\right\}+\beta^{i} D_{i}\left(c^{r k}\right) D_{k} u_{m},
\end{gathered}
$$

and $Q^{\prime}$ and $N^{\prime}$ are defined in $\S 1$. Hence Lemma 1 applies with all constants independent of $m$ and $r$. Therefore $|v|_{2+\alpha}$ is bounded independent of $m$ and $r$. It therefore follws that $\left|u_{m}\right|_{3+\alpha ; \partial \Omega}$ is bounded independent of $m$. The standard Schauder estimates [7, Problem 6.2] then give a uniform bound on $\left|u_{m}\right|_{3+\alpha}$ which then implies that $u \in C^{3+\alpha}(\bar{\Omega})$.

For operators in divergence form (as in $\S \S 4,5)$, the results of this section can be obtained more readily by other means. To do so, we introduce the following notation:

$$
\begin{array}{ll}
U(r)=\left\{x \in \mathbf{R}^{n}:|x|<r, x^{n}>0\right\}, & V(r)=\left\{x \in \mathbf{R}^{n}:|x| \leqslant r, x^{n} \geqslant 0\right\}, \\
F(r)=\left\{x \in \mathbf{R}^{n}:|x|<r, x^{n}=0\right\}, & G(r)=\left\{x \in \mathbf{R}^{n}:|x|<r, x^{n} \geqslant 0\right\}
\end{array}
$$

where $r$ is a positive number. We then have

LeMma $1^{\prime}$. Let $w \in C^{1}(V(r))$ satisfy the integral identity

$$
\int_{U(r)}\left\{a^{i j}(x) D_{j} w(x)+g^{i}(x)\right\} D_{i} \eta(x) d x=0
$$

for all $\eta \in C^{1}(V(r))$ with support in $G(r)$. Suppose that

$$
\beta \cdot D w=\psi \text { on } F(r) \text {. }
$$

If $\mathcal{Q}, \alpha$, and $\beta$ are as in Lemma 1 , then

$$
|w|_{1+\alpha ; V(r / 2)} \leqslant C(\alpha, \lambda, \Lambda, n, r)\left(|w|_{0 ; U(r)}+|g|_{\alpha ; U(r)}+|\psi|_{\alpha ; F(r)}\right) .
$$

Proof. The estimate is essentially a special case of [1, Theorem 9.3]. It may also be proved by a modification of the techniques in [7, Lemma 6.29].

From this result, we have a version of Lemma 2 with weaker regularity assumptions on $b$.

\section{Lemma 2'. Define}

$$
Q u=\operatorname{div}(A(x, u, D u))+B(x, u, D u), \quad N u=b(x, u, D u)
$$

and suppose that (2.2a) holds with

$$
a^{i j}(x, z, p)=\left(\partial A^{i} / \partial p_{j}\right)(x, z, p) .
$$

Suppose also that

$$
|b|_{1+\alpha ; K}+|A|_{1+\alpha ; K}+|B|_{\alpha ; K} \leqslant \Lambda
$$


where $\alpha, K, \lambda, \Lambda$ are positive constants with $\alpha<1$. Let $u \in C^{2+\alpha}(\bar{\Omega})$ be a solution of

$$
Q u=f \quad \text { in } \Omega, \quad N u=\varphi \quad \text { on } \partial \Omega
$$

such that $|u|_{1} \leqslant K$, and let $\delta \in(0,1)$. Then

$$
|u|_{2+\alpha} \leqslant C(\alpha, \delta, F, K, \lambda, \Lambda, U, \Phi, \Omega)
$$

where $F=|f|_{\alpha}, U=[D u]_{\delta}, \Phi=|\varphi|_{1+\alpha}$.

Proof. (In this proof $C$ denotes any constant depending only on $\alpha, \delta, F, K, \lambda, \Lambda, U, \Phi, \Omega$.)

It suffices to prove only that $|u|_{2+\alpha ; N} \leqslant C$ for some neighborhood $N$ of $\partial \Omega$. The global result then follows from interior Schauder estimates, e.g., [7, Corollary 6.3]. By a standard "straightening the boundary" argument we may assume that $u$ is a solution of $Q u=f$ in $U(1), N u=\varphi$ on $F(1)$ and that all hypotheses of this lemma are still valid with $\Omega$ replaced by $U(1)$ and $\partial \Omega$ replaced by $F(1)$. Then we need only show that $|u|_{2+\alpha ; U(1 / 4)} \leqslant C$.

In this case, we have (2.10) being satisfied where $w=D_{k} u$,

$$
g^{i}=D_{k} u A_{z}^{i}(x, u, D u)+A_{x^{k}}^{i}(x, u, D u)+\delta^{i k} B(x, u, D u)-\delta^{i k} f(x)
$$

and $\delta^{i k}$ is the Kronecker $\delta$. Also (2.11) holds with

$$
\beta=b_{p}(x, u, D u), \quad \psi=-D_{k} u b_{z}(x, u, D u)-b_{x^{k}}(x, u, D u)+D_{k} \varphi,
$$

provided $k \neq n$. A direct calculation shows that the hypotheses of Lemma $1^{\prime}$ hold with $r=1, \alpha=\alpha \delta$, and all constants independent of $k$. Thus

$$
\left|D_{i j} u\right|_{\alpha \delta} \leqslant C, \quad i=1, \ldots, n, j=1, \ldots, n-1 \text {. }
$$

Solving for $D_{n n} u$ in the equation $Q u=f$ then yields $\left|D^{2} u\right|_{\alpha \delta ; U(1 / 2)} \leqslant C$. Thus the hypotheses of this lemma hold with $\delta=1$. We then follow the above argument with $r=\frac{1}{2}$ to obtain the desired estimate.

We then have

LeMma $2^{\prime \prime}$. Define $Q$ and $N$ by (2.1)', and suppose that (2.2a) and (2.2b)' hold where $\alpha, K, \lambda, \Lambda$ are positive constants with $\alpha<1$. Suppose also that, for each positive integer $m$, there are $u_{m} \in C^{2+\alpha}(\bar{\Omega}), f_{m} \in C^{\alpha}(\bar{\Omega})$, and $\varphi_{m} \in C^{1+\alpha}(\partial \Omega)$ such that (2.3) and (2.4) hold with constants $K_{1}$ and $K_{2}$ independent of $M$. Then $u \in C^{2+\alpha}(\bar{\Omega})$ and $u$ is a solution of (2.5).

Analogs of Corollaries 1 and 2 are also valid with (2.2) replaced by (2.2a) and (2.2b)' in Corollary 1 .

3. Kirk and Caristi's Theorem. Our starting point is the following theorem of Caristi [2] (see also [4, Theorem 2] for a simple proof):

Lemma 3. Let $(V, d)$ be a complete metric space and let $g: V \rightarrow V$ be an arbitrary mapping such that

$$
d(v, g(v)) \leqslant \varphi(v)-\varphi(g(v)) \text { for all } v \in V
$$

where $\varphi: V \rightarrow \mathbf{R}^{+}$is a lower semicontinuous function. Then $g$ has a fixed point. 
To apply this lemma, we first need a definition. Let $X$ and $Y$ be Banach spaces and $P: X \rightarrow Y$ a mapping such that

$$
P_{x}(\psi) \equiv \lim _{\varepsilon \rightarrow 0} \frac{P(x+\varepsilon \psi)-P(x)}{\varepsilon}
$$

exists for all $x$ and $\psi$ in $X$. Then we say that $P_{x}$ is the Gateaux variation of $P$.

Lemma 4. Let $P: X \rightarrow Y$ have a Gateaux variation and suppose that $P(X)$ is closed in $Y$. If, for all $x \in X$, there is $\psi \in X$ such that $P_{x}(\psi)+P x=0$, then $0 \in P(X)$.

Proof. For each $y \in P(X)$, choose $x \in X$ and $\psi \in X$ such that $y=P x, P_{x}(\psi)+$ $P x=0$. By the definition of $P_{x}$, there exists $\varepsilon>0$ such that

$$
\left\|(P(x+\varepsilon \psi)-P x)-\varepsilon P_{x}(\psi)\right\| / \varepsilon \leqslant\|P x\| / 2
$$

where \|\| is the norm in $Y$. Then

$$
(\varepsilon / 2)\|P x\| \geqslant\|P(x+\varepsilon \psi)-(1-\varepsilon)(P x)\| .
$$

The triangle inequality then implies that

$$
\|(P(x+\varepsilon \psi)-(P x))\| \leqslant(3 \varepsilon / 2)\|P x\|, \quad(\varepsilon / 2)\|P x\| \leqslant\|P x\|-\|P(x+\varepsilon \psi)\|,
$$

and hence that

$$
\|(P(x+\varepsilon \psi)-P x)\| \leqslant 3(\|P x\|-\|P(x+\varepsilon \psi)\|) .
$$

We now make the identifications $V=P(X), d\left(v_{1}, v_{2}\right)=\left\|v_{1}-v_{2}\right\|, \varphi(v)=3\|v\|$ in Lemma 3 and define $g(y)=P(x+\varepsilon \psi)$. Since $V$ is complete, $g$ has a fixed point which must be zero by (3.1). Therefore $0=P x$ for some $x \in X$.

A slight modification of the proof of Lemma 4 gives a result which we shall use in $\S 5$.

Lemma 4'. Let $P: X \rightarrow Y$ have a Gateaux variation and let $Y_{0}$ be a subset of $Y$. Suppose that $Y_{0} \cap P(X)$ is closed. Suppose also that

(3.2a) $0 \in Y_{0}$,

(3.2b) $Y_{0} \cap P(X)$ is nonempty,

(3.2c) for all $x \in P^{-1}\left(Y_{0}\right)$ and $\psi \in X$ such that $P_{x}(\psi)+P x=0$, we have $P(X+\varepsilon \psi) \in Y_{0}$ for all sufficiently small $\varepsilon$.

If, for all $x \in P^{-1}\left(Y_{0}\right)$, there is $\psi \in X$ such that $P_{x}(\psi)+P x=0$, then $0 \in P(X)$.

Remarks. (1) Lemma 4 is implicit in the work of Kirk and Caristi (cf. [8]); the above formulation and proof are from a personal communication from $\mathrm{W}$. O. Ray.

(2) The quantity $\varepsilon / 2$ in $(3.1)$ may be replaced by $q \varepsilon$ where $q \in(0,1)$ is arbitrary but independent of $y$.

(3) If (3.1) with $q \varepsilon$ can be proved without the assumption that $P_{x}$ exists, then it still follows that $0 \in P(X)$.

(4) When using Caccioppoli's theorem, a similar circumstance arises (see [5, §7]). In this case, the existence of the Fréchet derivative of $P$ is used to invoke a theorem of Hildebrandt and Graves (see [11, §41]). 
To apply Lemma 4, we define $Q$ and $N$ by (2.1) and suppose that for constant $\alpha \in(0,1)$ and all $K>0$ the conditions $(2.2 \mathrm{a})$ and

$$
\partial \Omega \in C^{3+\alpha}, \quad|Q|_{2+\alpha ; K}+|a|_{2+\alpha ; K}+|b|_{3+\alpha ; K} \leqslant \Lambda
$$

are satisfied with $\lambda, \Lambda$ positive constants possibly depending on $K$. For each $\beta \in(0, \alpha)$ we define

$$
P: C^{3+\beta}(\bar{\Omega}) \rightarrow C^{1+\beta}(\Omega)
$$

by

$$
P u=(Q u, N u)
$$

where, for any $\delta>0$,

$$
\bigodot^{\delta}(\Omega)=C^{\delta}(\bar{\Omega}) \times C^{1+\delta}(\delta \Omega) .
$$

Then the Gateaux variation of $P$ exists and

$$
P_{u}(\psi)=\left(Q_{u}(\psi), N_{u}(\psi)\right)
$$

where

$$
\begin{aligned}
& Q_{u}(\psi)=a^{i j}(x, u, D u) D_{i j} \psi+Q^{\prime}\left[u ; p_{k}\right] D_{k} \psi+Q^{\prime}[u ; z] \psi, \\
& N_{u}(\psi)=N^{\prime}\left[u ; P_{k}\right] D_{k} \psi+N^{\prime}[u ; z] \psi .
\end{aligned}
$$

We then have our main theorem.

Theorem 1. Define $Q$ and $N$ be (2.1). Suppose that conditions (2.2a) and (3.3) are satisfied and define $P$ by (3.4). Suppose also that for all $(f, \varphi) \in C^{1+\beta}(\Omega)$ and $u \in C^{3+\beta}(\bar{\Omega})$ such that $P u=(f, \varphi)$, the estimate

$$
|u|_{1+\delta} \leqslant K(Q, N, F, \Phi, \Omega) \text { for some } \delta \in(0,1)
$$

is valid with $F=|f|_{1+\beta}, \Phi=|\varphi|_{2+\beta}$. Suppose finally that

(3.6) for all $u \in C^{3+\beta}(\bar{\Omega})$, there exists $\psi \in C^{3+\beta}(\bar{\Omega})$ such that $P_{u}(\psi)+P u=0$.

Then the problem

$$
Q u=0 \quad \text { in } \Omega, \quad N u=0 \text { on } \partial \Omega
$$

has a solution $u \in C^{3+\beta}(\bar{\Omega})$.

RemarKs. (1) The space $e^{1+\beta}(\Omega)$ may be replaced by any subset $Y_{0}$ satisfying (3.2).

(2) The problem (3.7) is still solvable with $u \in C^{2+\beta}$ if $\mathscr{Q}$ and $a$ are only Hölder-continuous provided (3.5) and (3.6) hold uniformly for an approximating sequence of operators. Here we may assume $F=\Phi=0$ in (3.5).

(3) Since $P_{u}(\psi)+P u=0$ is a linear boundary value problem for $\psi$, condition (3.6) follows from the conditions

$$
\partial a^{i j} / \partial z=0, \quad \partial a / \partial z<0, \quad \partial b / \partial z \leqslant 0 .
$$

(See [7, Theorem 6.31] or [9, §3.3].)

(4) If the estimate (3.5) holds with $F=|f|_{\beta}, \Phi=|\varphi|_{1+\beta}$, then a different version of Theorem 1 can be proved (see Theorem 1' below) without using Corollaries 1 and 2. 
THEOREM 1'. Define $Q$ and $N$ by (2.1). Suppose that for some $\alpha \in(0,1)$ and all $K>0$, conditions (2.2) are fulfilled with $\lambda$ and $\Lambda$ depending on $K$. Define $P$ : $C^{2+\beta}(\bar{\Omega}) \rightarrow \bigodot^{\beta}(\Omega)$ for $\beta \in(0, \alpha)$ by (3.4) and suppose that (3.5) holds with $F=|f|_{\beta}$, $\Phi=|\varphi|_{1+\beta}$. Suppose finally that

(3.6)" for all $u \in C^{2+\beta}(\bar{\Omega})$, there exists $\psi \in C^{2+\beta}(\bar{\Omega})$ such that $P_{u}(\psi)+P u=0$.

Then problem (3.7) has a solution $u \in C^{2+\beta}(\bar{\Omega})$.

Theorem 1 and $1^{\prime}$ show that the crucial steps in solving (3.7) are establishing (3.5) and (3.6) or (3.6)". The following two sections discuss two examples for which these conditions have been obtained.

4. Uniformly elliptic equations. We now consider the problem of uniformly elliptic equations as in $[9, \S 10.2]$. Specifically

$$
\begin{aligned}
& Q u=\operatorname{div}(A(x, u, D u))+B(x, u, D u), \\
& N u=A^{i}(x, u, D u) \gamma_{i}+\psi(x, u) .
\end{aligned}
$$

We briefly list the results of Ladyzhenskaya and Ural'ceva from $[9, \S 10.2]$.

Lemma 5. Define $Q$ and $N$ by (4.1), and suppose that

(4.2a) $\left(\partial A^{i} / \partial p_{j}\right)(x, z, p) \xi_{i} \xi_{j} \geqslant 0$ for $x \in \partial \Omega, p \in \mathbf{R}^{n}$ or $x \in \Omega, p=0$,

$$
\begin{gathered}
z\left[\left(\partial A^{i} / \partial x^{i}\right)(x, z, 0)+B(x, z, 0)-f(x)\right]<0 \quad \text { for } x \in \Omega, \\
z\left[A^{i}(x, z, 0) \gamma_{i}-\varphi(x)+\psi(x, z)\right]<0 \quad \text { for } x \in \partial \Omega
\end{gathered}
$$

whenever $|z| \geqslant R$ where $\lambda$ and $R$ are positive constants. Then any solution $u \in C^{1}(\bar{\Omega})$ $\cap C^{2}(\Omega)$ of $(2.5)$ is bounded by $|u|_{0} \leqslant R$.

Proof. [9, Lemma 2.1, Chapter 10].

Lemma 6. Define $Q$ and $N$ by (4.1) and let $u \in C^{2}(\bar{\Omega})$ be a solution of (2.5) with $|u|_{0} \leqslant M$, some positive constant. Suppose also that

$$
\begin{aligned}
\left(\partial A^{i} / \partial p_{j}\right)(x, z, p) \xi_{i} \xi_{j} & \geqslant \nu(1+|p|)^{m-2}|\xi|^{2}, \\
|A(x, z, p)|+\left|A_{x}(x, z, p)\right| & +\left|A_{z}(x, z, p)\right| \\
& +(1+|p|)\left|A_{p}(x, z, p)\right| \leqslant \mu(1+|p|)^{m-1},
\end{aligned}
$$

for all $(x, z, p) \in \Omega \times \mathbf{R} \times \mathbf{R}^{n}$ with $|z| \leqslant M$, where $m, \mu, \nu$ are positive constants with $m>1$. Then

$$
|\tilde{D} u|_{0} \leqslant K_{0}(F, m, M, \mu, \nu, \Phi, \Psi, \Omega)
$$

where $F=|f|_{0}, \Phi=|\varphi|_{1}, \Psi=|\psi|_{1 ; M}$.

Proof. [9, Theorem 2.1, Chapter 10 (see especially pp. 468-476)].

The hypotheses (4.3) are actually somewhat weaker than those in [9], but the same proof is still valid in this case. (This observation is made in $[10, \S \mathrm{V} .7]$.) 
LEMma 7. Define $Q$ and $N$ by (4.1) and let $u \in C^{2}(\bar{\Omega})$ be a solution of (2.5) with $|u|_{1} \leqslant K_{0}$. Suppose that

$$
\begin{gathered}
\mu|\xi|^{2} \geqslant\left(\partial A^{i} / \partial p_{j}\right)(x, z, p) \xi_{i} \xi_{j} \geqslant \nu|\xi|^{2} \quad \text { for all }(x, z, p) \in \Omega\left(K_{0}\right), \\
|A|_{1 ; K_{0}}+|B|_{0 ; K_{0}}+|\varphi|_{1}+|f|_{0}+|\psi|_{1 ; K_{0}} \leqslant \mu
\end{gathered}
$$

where $\mu$ and $\nu$ are positive constants. Then, for some positive constants $\delta$ and $K$ depending only on $K_{0}, \mu, \nu, \Omega$, we have the estimate $|u|_{1+\delta} \leqslant K$.

Proof. [9, Theorem 2.1, Chapter 10 (see especially pp. 467-468)].

LEMMA 8. Define $Q$ and $N$ by (4.1) and suppose that $u \in C^{2+\alpha}(\bar{\Omega})$ is a solution of (2.5) with $|u|_{1} \leqslant K_{0}$ for some $\alpha \in(0,1)$. Suppose also that (4.4) holds for all $(x, z, p) \in \Omega\left(K_{0}\right)$ where $\mu, \nu$ are positive constants and that $A \in C^{2+\alpha}\left(\bar{\Omega}\left(K_{0}\right)\right)$, $B \in C^{1+\alpha}\left(\bar{\Omega}\left(K_{0}\right)\right), \psi \in C^{1+\alpha}\left(\partial \Omega\left(K_{0}\right)\right)$. Suppose finally that

$$
\begin{gathered}
\psi_{z}(x, z) \leqslant 0, \\
B_{z}(x, z, p)+\left(\left|A_{z}(x, z, p)\right|^{2}+\left|B_{p}(x, z, p)\right|^{2}\right) / \nu<0
\end{gathered}
$$

for all $x \in \Omega, z=u, p=D u$. Then $P_{u}: C^{2+\beta}(\bar{\Omega}) \rightarrow \bigodot^{\beta}(\Omega)$ is surjective for all $\beta \in(0, \alpha)$ where $P$ and $C$ are defined in (3.4).

Proof. [9, Lemma 2.3, Chapter 10].

Combining these lemmata with Theorem 1' yields an existence result (cf. [9, Theorem 2.2, Chapter 10]).

THEOREM 2. Let $Q$ and $N$ be defined by (4.1) and suppose that conditions (4.2b) and (4.2c) hold for sufficiently large $z$ and all $p \in \mathbf{R}^{n}$. Suppose that (4.3) holds for all $(x, z, p) \in \bar{\Omega} \times \mathbf{R} \times \mathbf{R}^{n}$ with $m>1$ a constant, $\mu$ an increasing function of $|z|, \nu a$ decreasing function of $|z|$, and $\nu(t)>0$ for all $t>0$. Suppose that (4.6) holds for all $(x, z, p) \in \bar{\Omega} \times \mathbf{R} \times \mathbf{R}^{n}$ and that

$$
\begin{gathered}
A \in C^{2+\alpha}\left(\bar{\Omega} \times \mathbf{R} \times \mathbf{R}^{n}\right), \quad B \in C^{2+\alpha}\left(\bar{\Omega} \times \mathbf{R} \times \mathbf{R}^{n}\right), \\
\psi \in C^{1+\alpha}(\partial \Omega \times \mathbf{R})
\end{gathered}
$$

for some $\alpha>0$. Then for any $\beta \in(0, \alpha)$, there is a solution $u \in C^{2+\beta}(\bar{\Omega})$ of

$$
Q u=0 \quad \text { in } \Omega, \quad N u=0 \text { on } \partial \Omega .
$$

REMARK. From Lemma $1^{\prime}$, it follows that this solution $u$ is in $C^{2+\alpha}(\bar{\Omega})$. Moreover if all the hypotheses of Theorem 2 are satisfied except that $A$ is only $C^{1+\alpha}$ and $B$ is only $C^{1}$, then an approximation argument can be used to show that there is a solution $u \in C^{2+\alpha}(\bar{\Omega})$ to $(4.8)$.

5. The capillarity problem. We consider here the problem

$$
\operatorname{div}(T u)=\kappa u \quad \text { in } \Omega, \quad T u \cdot \gamma=g(x) \text { on } \partial \Omega
$$

where $T u=D u / W, W=\left(1+|D u|^{2}\right)^{1 / 2}$ and $\kappa$ is a positive constant. When $n=2$, a solution $u$ of (5.1) describes the height of liquid in a capillary tube.

To write (5.1) in a form consistent with previous sections, we set

$$
Q u=\operatorname{div}(T u)-\kappa u=a^{i j} D_{i j} u+a, \quad N u=T u \cdot \gamma-g,
$$




$$
a^{i j}=\left(\delta^{i j} W^{2}-D_{i} u D_{j} u\right) / W^{3} ; \quad a=-\kappa u,
$$

where $\delta^{i j}$ is the Kronecker $\delta$ and consider solutions of

$$
Q u=f \quad \text { in } \Omega, \quad N u=\varphi \quad \text { on } \partial \Omega .
$$

Estimates for solutions of (5.3) are well known; we present them below.

LEMMA 9. If $u \in C^{2}(\bar{\Omega})$ is a solution of (5.3) and

$$
\sup _{\partial \Omega}|\varphi+g| \leqslant 1-d \text { where } d \in(0,1) \text { is constant, }
$$

then $|u|_{0} \leqslant K_{0}(d, F, \kappa, \Omega)$ where $F=|f|_{1}$.

PROOF. The corollary on p. 208 of [3].

LEMMA 10. If $u \in C^{2}(\bar{\Omega})$ is a solution of (5.3), and if (5.4) holds, then $|D u|_{0} \leqslant$ $K_{0}(d, F, \kappa, M, \Phi, \Omega)$ where $F=|f|_{1}, \Phi=|\varphi|_{1}$ and $M=|u|_{0}$.

Proof. [6, Theorem 1.1], which is a modification of the proof in [14]. Another proof under somewhat different hypotheses is given in [13].

From these lemmata and Remark (1) after Theorem 1, we can prove solvability of (5.1).

THEOREM 3. If $|g|_{0 ; \partial \Omega} \leqslant 1-d$ and $g \in C^{1+\alpha}(\partial \Omega)$, then there exists a solution $u \in C^{2+\alpha}(\bar{\Omega})$ of $(5.1)$.

Proof. Suppose first that $g \in C^{2+\alpha}(\partial \Omega)$. We define $X=C^{2+\alpha}(\bar{\Omega}), Y=e^{1+\alpha}(\Omega)$,

$$
Y_{0}=\left\{(f, \varphi) \in \mathrm{e}^{1+\alpha}(\Omega): \sup _{\partial \Omega}|\varphi+g| \leqslant 1-(d / 2)\right\} .
$$

From Remark (3) after Theorem 1, it follows that $P_{u}$ is surjective. Condition (3.2a) is easily verified as is (3.2b). To check (3.2c), we note that if $P_{u}(\psi)+P u=0$ and $\varepsilon$ is sufficiently small, then, as in Lemma 4 , we have

$$
\|P(u+\varepsilon \psi)-(1-\varepsilon) P u\| \leqslant \varepsilon d / 2 .
$$

The inequalities $\|(f, \varphi)\| \geqslant \sup _{\partial \Omega}|\varphi|$ and the triangle inequality then give

$$
\sup _{\partial \Omega}|N(u+\varepsilon \psi)+g| \leqslant \varepsilon(1-d)+\varepsilon(d / 2)+(1-\varepsilon)(1-(d / 2))=1-(d / 2)
$$

provided $u \in P^{-1}\left(Y_{0}\right)$. Lemmata 7, 9, 10 and Theorem 1 imply that $0 \in P(X)$ which means that (5.1) has a solution in $C^{3+\alpha}(\bar{\Omega})$.

Now suppose that $g \in C^{1+\alpha}(\partial \Omega)$ and let $\left(g_{m}\right)$ be a sequence in $C^{2+\alpha}(\partial \Omega)$ converging uniformly to $g$ and such that

$$
\left|g_{m}\right|_{1+\alpha} \leqslant C|g|_{1+\alpha} \text { for each } m
$$

where $C$ is independent of $m$. By what we have already proved, there exists $u_{m}$ in $C^{3+\alpha}(\bar{\Omega})$ such that $Q u_{m}=0$ in $\Omega, T u_{m} \cdot \gamma=g_{m}$ on $\partial \Omega$. Inequality (5.5) in conjunction with Lemmata 7,9 , and 10 implies that, for some $\delta \in(0,1)$, the norms $\left|u_{m}\right|_{1+\delta}$ are bounded uniformly with respect to $m$. The Arzela-Ascoli theorem implies that some subsequence of $\left(u_{m}\right)$ converges uniformly to a function $u$. Applying Lemma 2 to this subsequence, we infer that $u \in C^{2+\alpha}(\bar{\Omega})$ and that $u$ is a solution of (5.1). 
REMARKs. (1) Theorem 3 is well known, but ours is the first proof that does not rely on the existence of solutions to other nonlinear boundary-value problems (cf. $[6,13,14])$.

(2) Theorem 3 can also be proved without using Remark (1) after Theorem 1 by defining $N u=\tanh ^{-1}(T u \cdot \gamma)$ in (5.2).

\section{REFERENCES}

1. S. Agmon, A. Douglis and L. Nirenberg, Estimates near the boundary for solutions of elliptic partial differential equations satisfying general boundary conditions. I, Comm. Pure Appl. Math. 12 (1959), 623-727.

2. J. Caristi, Fixed point theorems for mappings satisfying inwardness conditions, Trans. Amer. Math. Soc. 215 (1976), 241-251.

3. P. Concus and R. Finn, On capillary free surfaces in a gravitational field, Acta Math. 132 (1974), 207-223.

4. I. Ekeland, Nonconvex minimization problems, Bull. Amer. Math. Soc. (N. S.) 1 (1979), 443-474.

5. R. Fiorenza, Sui problemi di derivata obliqua per le equazioni ellittiche, Ricerche Mat. 8 (1959), 83-110.

6. C. Gerhardt, Global regularity of the solutions to the capillarity problem, Ann. Scuola Norm. Sup. Pisa Cl. Sci. (4) 3 (1976), 157-176.

7. D. Gilbarg and N. S. Trudinger, Elliptic partial differential equations of second order, Die Grundlehren der math. Wissenschaften, Band 224, Springer-Verlag, Berlin and New York, 1977.

8. W. A. Kirk and J. Caristi, Mapping theorems in metric and Banach spaces, Bull. Acad. Polon. Sci. Sér. Sci. Math. Astronom. Phys. 23 (1975), 891-894.

9. O. A. Ladyzhenskaya and N. N. Ural'ceva, Linear and quasilinear elliptic equations, Mathematics in Science and Engineering, 46, Academic Press, New York, 1968.

10. O. A. Ladyzenskaja, V. A. Solonnikov and N. N. Ural'ceva, Linear and quasilinear equations of parabolic type, Transl. Math. Mono., vol. 23, Amer. Math. Soc., Providence, R. I., 1968.

11. C. Miranda, Partial differential equations of elliptic type, Ergebnisse der Math. Grenzgebiete, Band 2, 2nd ed., Springer-Verlag, Berlin and New York, 1970.

12. J. Serrin, The problem of Dirichlet for quasilinear elliptic equations with many independent variables, Philos. Trans. Roy. Soc. London Ser. A 264 (1969), 413-496.

13. L. Simon and J. Spruck, Existence and regularity of a capillary surface with prescribed contact angle, Arch. Rational. Mech. Anal. 61 (1976), 19-34.

14. N. N. Ural'ceva, Solvability of the capillary problem, Vestnik Leningrad Univ. Math. 6 (1979), $363-375$.

Department of Mathematics, Iowa State University, Ames, Iowa 50011 